\title{
Identifying Six Novel Genetic Variants and Alterations in Peripheral T-cell Subsets in Porokeratosis Patients
}

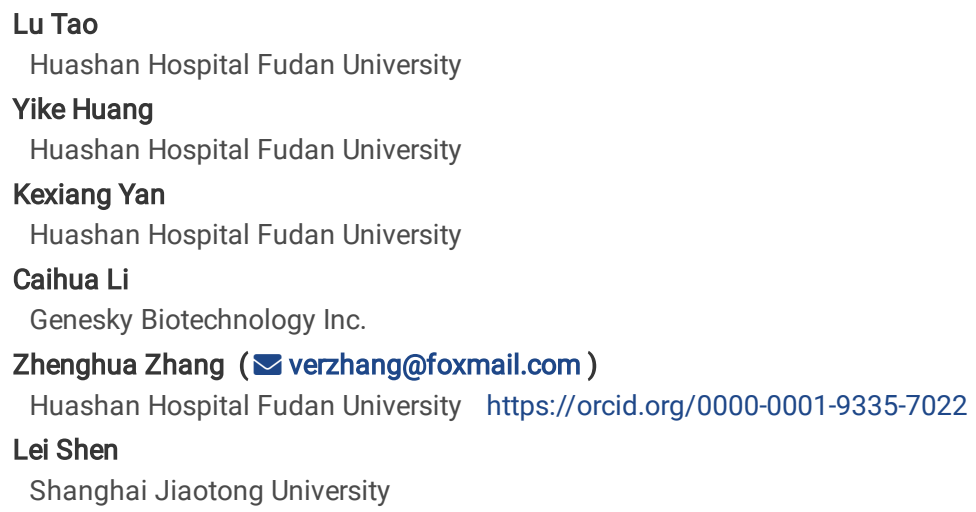

\section{Short report}

Keywords: porokeratosis, autoinflammation, MVK, MVD

Posted Date: July 6th, 2021

DOI: https://doi.org/10.21203/rs.3.rs-632232/v1

License: (c) (1) This work is licensed under a Creative Commons Attribution 4.0 International License. Read Full License 


\begin{abstract}
Porokeratosis (PK) is considered a skin-specific autoinflammatory keratinization disease. Intriguingly, four causative genes of PK are in turn arranged in mevalonate pathway, with MVD variants being the commonest followed by MVK variants in a cohort of Chinese patients. Based on our previous findings, PK patients with $M V K$ or $M V D$ variants show different phenotypes. Using targeted exome sequencing and exonic CNV screening, we identified 14 mutations in the 26 PK patients, including six novel mutations (MVK: c.118_226 + 1337dup, c.388_392delGATATinsC, c.613A > T, c.768G > C, and MVD: c.250C > T, c.988T > G). Peripheral T-cell subsets were analyzed by flow cytometry in PBMCs from 22 PK patients with MVK or MVD variants and 27 normal controls (NCs). In contrast to NCs, significantly decreased frequencies of CD8 ${ }^{+}$and Vy9Vס2 T cells were observed in the PK patients with MVD variants. Moreover, it was found that dysregulated secretion of pro-inflammatory cytokines by T-cell in both PK patients with MVK and MVD variants. Collectively, our findings enriched the Human Gene Mutation Databases and provided the cues to further studies on autoreactive CD8 $+\mathrm{T}$ and $\gamma \delta T$ cells in the pathogenesis of PK.
\end{abstract}

\title{
Introduction
}

As a skin-specific autoinflammatory keratinization diseases(AlKDs), porokeratosis (PK, MIM 175800) is inherited in an autosomal dominant pattern, whose genetic causative factors are associated with the hyperactivation of innate immunity, mainly in the epidermis and upper dermis [1-3]. It is known that the four causative genes of PK are in turn arranged in mevalonate pathway, i.e. mevalonate kinase ( $M V K)$, phosphomevalonate kinase ( $P M V K)$, mevalonate (diphospho) decarboxylase (MVD), and farnesyl diphosphate synthase (FDPS) [4,5]. Among them, MVD variants are the commonest cause followed by MVK variants in a cohort of Chinese patients [5-7]. Notably, the patients with MVK variants generally showed the widest range of phenotypes in terms of both the number and the size of lesions. Giant plaque-type PK ptychotropica (PPt) appears to be a unique phenotype associated with $M V K$ variants. The lesions of $M V D$ variants tend to be more homogeneous and superficial than those carrying MVK variants. The possible reason is that MVK and MVD deficiencies result in different metabolites of the mevalonate pathway. Generally, it is accepted that mevalonate metabolites regulate T-cell at multiple levels [8, 9]. Based on the previous findings, we hypothesized that T-cell bearing heterozygous mutations in mevalonate pathway might be altered and involved in the autoinflammation of PK. In this study, we preliminarily analyzed the distribution and cytokine production of peripheral T-cell subsets in PK patients with MVK or MVD genotype.

\section{Materials And Methods Clinical Samples}

All procedures followed the guidelines of the Helsinki Declaration and were approved by the Scientific Ethical Committee of Fudan University. Study participants provided informed consent for genetic testing and flow cytometry. From 2018 to 2019 , peripheral blood samples were collected from 26 patients with PK (13 males and 13 females; mean age $53 \pm 3$ years). Among them, there were 3 patients form one family. In addition, blood samples were collected from 27 healthy adult individuals ( 9 males and 18 females; mean age $42 \pm 2$ years). The 26 patients were diagnosed by at least two experienced dermatologists, based on both clinical features and histological examinations. In view of the family history, these patients were divided into 15 familial and 9 sporadic cases.

\section{Targeted exome sequencing and exonic CNV screening}

Total DNA was extracted from the peripheral blood using a QIAamp DNA Blood Mini Kit (Qiagen, Germany). Targeted exome sequencing and CNVplex assays were performed as previously described [5]. The sequenced reads were collected, filtered for quality, and aligned to the human genome reference assembly (UCSC Genome Browser hg19; https://genome.ucsc.edu/index.html) with the Burrows-Wheeler Aligner. Variants with minor allele frequency of $>0.05$ in any of the variant databases were excluded. SIFT, PolyPhen-2, and MutationTaster were used to assess the conservation of the missense mutations. Nonsynonymous SNVs with SIFT scores $<0.05$, Polyphen-2 scores $>0.85$, or MutationTaster scores $>0.85$ were considered as significant of not being benign.

\section{Flow cytometry and Statistical analysis}

Peripheral blood mononuclear cells (PBMCs) were isolated from heparinized blood using Ficoll-Hypaque (Lymphoprep; Stemcell Technologies) by density gradient centrifugation and stored frozen in liquid nitrogen until use. To evaluate cytokine production ex vivo, PBMCs were rested overnight and then stimulated for 5 hours phorbol 12-myristate 13-acetate (PMA; $50 \mathrm{ng} / \mathrm{ml}$; Sigma) and ionomycin (2 $\mu \mathrm{g} / \mathrm{ml}$; Enzo Life Sciences). Brefeldin A (10 $\mu \mathrm{g} / \mathrm{ml}$; BioLegend) was present during the final 2 hours. PBMCs were first stained for 15 minutes with Live/Dead Blue (Invitrogen) to exclude dead cells and then stained for 30 minutes with APC-Cy7-conjugated anti-human CD3 (clone SK7; BD Biosciences), BV786-conjugated anti-human CD8 (clone RPA-T8; BD

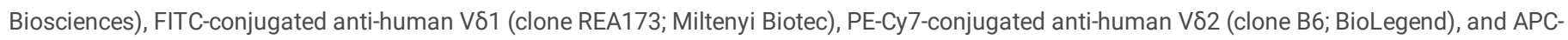
conjugated anti-human VY9 (clone B3; BioLegend). The cells were then washed, fixed, and permeabilized for 40 minutes using a BD Cytofix/Cytoperm Kit (BD Biosciences). Finally, the cells were intracellularly stained for 40 minutes with BV421-conjugated anti-human TNF-a (clone MAb11; BD Biosciences) and PEconjugated anti-human IFN- $\gamma$ (clone 4S.B3; BD Biosciences). Data were acquired on a BD LSRFortessa ${ }^{\text {TM }}$ X-20 flow cytometer (BD Biosciences) and analyzed with FlowJo software (Tree Star Inc.) Data analyses and graph preparation were performed using GraphPad Prism v.8 (GraphPad Software, San Diego, CA, U.S.A.). The data were expressed as mean \pm standard error of the mean (SEM). Statistical analysis was performed using one-way analysis of variance (ANOVA) with $p$-values $<0.05$ being considered statistically significant.

\section{Results}




\section{Identification of six novel mutations in MVK and MVD genes}

Table 1 provided a detailed description and characterization of 14 variants identified in this study. One novel tandem duplication (c.118_226 + 1337dup) in MVK was found in Fig. 1 by CNVplex assay. Besides, Fig. 2 presented three novel variants in MVK (c.388_392delGATATinsC, c.613A > T, c.768G >C) and two novel variants in $M V D$ (c. $250 \mathrm{C}>\mathrm{T}$, c. $988 \mathrm{~T}>\mathrm{G}$ ). The commonest $M V D$ mutation (c.746T > C) was identified in seven of the unrelated patients, accounting for $26.9 \%$ of $26 \mathrm{PK}$ patients. No mutation was found in 3 sporadic PK patients. Among them, 12 patients with MVD variants were selected to compare with 10 patients with $M V K$ variants.

Table 1

Characterization of 14 variants identified in 21 of the 24 PK index patients.

\begin{tabular}{|c|c|c|c|c|c|c|c|c|c|c|}
\hline No. & Gene & Mutation & Exon & $\begin{array}{l}\text { Predicted protein } \\
\text { altemation }\end{array}$ & $\begin{array}{l}\text { Mutation } \\
\text { type }\end{array}$ & $\begin{array}{l}\text { SIFT } \\
\text { Score }\end{array}$ & $\begin{array}{l}\text { POLY } \\
\text {-PHEN } \\
\text { Score }\end{array}$ & $\begin{array}{l}\text { MutationTaster } \\
\text { Score }\end{array}$ & $\begin{array}{l}\text { ExAc_ } \\
\text { EAS }\end{array}$ & $\begin{array}{l}\text { GenomeAD_ } \\
\text { Exomes_EAS }\end{array}$ \\
\hline \multirow[t]{2}{*}{1} & $M V K$ & c.118_226+1337dup & 2,3 & p.? & Tandem & & & & & \\
\hline & & & & & Duplication & & & & & \\
\hline \multirow[t]{2}{*}{2} & $M V K$ & c.388_392delGATATinsC & 5 & p.Asp130Profs*2 & Frameshift & & & & & \\
\hline & & & & & Substitution & & & & & \\
\hline 3 & $M V K$ & c. $451 \mathrm{G}>\mathrm{A}$ & 5 & p.Val151Met & Missense & 0.01 & 0.938 & 0.999995 & & \\
\hline 4 & $M V K$ & c. $613 \mathrm{~A}>\mathrm{T}$ & & p.Asn205Tyr & Missense & 0 & 1 & 1 & & \\
\hline 5 & $M V K$ & c. $710 \mathrm{C}>\mathrm{A}$ & 8 & p.Thr237Asn & Missense & 0 & 0.992 & 0.999254 & & \\
\hline 6 & $M V K$ & c. $768 \mathrm{G}>\mathrm{C}$ & 8 & p.Lys256Asn & Missense & 0.005 & 0.86 & 1 & & \\
\hline 7 & $M V K$ & c. $1039+2 \mathrm{~T}>\mathrm{C}$ & 10 & p.? & Splice_Site & & & 1 & & \\
\hline 8 & $M V K$ & c. $1126 \mathrm{G}>\mathrm{A}$ & 11 & p.Gly376Ser & Missense & 0 & 0.996 & 0.999999 & & \\
\hline 9 & $P M V K$ & c. $412 \mathrm{C}>\mathrm{T}$ & 4 & p.Arg138* & Nonsense & 1 & 0.735406 & 1 & & \\
\hline 10 & $M V D$ & c. $250 \mathrm{C}>\mathrm{T}$ & 3 & p.Arg84Trp & Missense & 0.008 & 0.97 & 0.987 & 0.0002 & 0.0002 \\
\hline 11 & $M V D$ & c. $383 \mathrm{C}>\mathrm{T}$ & 4 & p.Ala128Val & Missense & 0 & 0.998 & 0.999994 & 0.0002 & 0.0001 \\
\hline 12 & $M V D$ & c. $746 \mathrm{~T}>\mathrm{C}$ & 7 & p.Phe249Ser & Missense & 0 & 1 & 0.999989 & 0.0005 & 0.0002 \\
\hline 13 & $M V D$ & c. $988 \mathrm{~T}>\mathrm{G}$ & 8 & p.Phe330Val & Missense & 0.001 & 0.009 & 1 & & \\
\hline 14 & $M V D$ & c.1111_1113del & 9 & p.lle371del & $\begin{array}{l}\text { In-Frame } \\
\text { Deletion }\end{array}$ & & & & 0.0002 & 0.0001 \\
\hline
\end{tabular}

Notes:

1. As for the familial cases, the number of affected and unaffected members were listed in parentheses. SIFT, PolyPhen-2, and MutationTaster were used to a missense mutations.

2. ExAC_EAS:Exome Aggregation Consortium_East Asian allele frequency; GnomeAD_Exomes_EAS : The Genome Aggregation Database_Exomes_East Asiar

\section{Decreased frequencies of CD8 ${ }^{+}$and Vy9Vס2 T-cell in the PK patients with MVD variants}

As indicated in Fig. 3, the PK patients with $M V D$ variants exhibited a significant decrease in the frequencies of peripheral CD8 ${ }^{+}$and $V y 9 V 82 \mathrm{~T}^{-c e l l}$ in the CD3 ${ }^{+} \mathrm{T}-$ cell subsets compared with that of the NCs $(p=0.0009$ and $p=0.0216$, respectively). Therefore, the PK patients with $M V D$ variants had a correspondingly

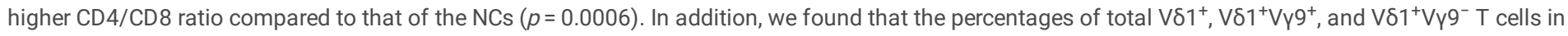
the $\mathrm{CD}^{+} \mathrm{T}$-cell subsets remained unchanged in the PK patients with either MVK or MVD variants compared with that of the NCs (Additional file 1: Figure S1).

\section{Dysregulated secretion of pro-inflammatory cytokines by T-cell in the PK patients with MVK or MVD variants}


As indicated in Fig. 4, IFN-y production by $\mathrm{CD} 8^{+}$T-cell was increased in PK patients with MVK variants compared with that of the NCs and PK patients with $M V D$ variants ( $p=0.0275$ and $p=0.0301$, respectively). Moreover, TNF-a production by VY9V $\delta 2$ T-cell was significantly increased in the PK patients with MVD variants compared with that of the NCs and the PK patients with MVK variants ( $p=0.0085$ and $p=0.0432$, respectively).

\section{Discussion}

In this study, 22 PK patients with MVK or MVD variants were identified by targeted exome sequencing and exonic CNV screening. The distribution and cytokine production of peripheral T cells subsets were preliminary analyzed in those patients. It is well known that peripheral a $\mathrm{T}$ cells are primarily comprised of CD $4^{+}$ $T$ cells and $C D 8^{+} T$ cells. Instead of directly staining CD4, we counted all $C D 3^{+} V \delta 1^{-} V \delta 2^{-} C D 8^{-}$cells as $C D 4^{+} T$ cells. Besides the frequency of $T$ cells subsets, we assessed the pro-inflammatory cytokines production by CD4 ${ }^{+} \mathrm{T}$ cells, CD8 ${ }^{+} \mathrm{T}$ cells, and Vy9V82 $\mathrm{T}$ cells in the peripheral blood. It was considered that the increases in percentages of peripheral $\mathrm{CD} 4^{+} \mathrm{T}$ cells were a secondary change, since the cytokines production by $\mathrm{CD} 4^{+} \mathrm{T}$ cells were unchanged in the $\mathrm{PK}$ patients with either $M V K$ or $M V D$ variants.

Different from the patients with $M V K$ variants, we observed that there were significant decreases in percentages of both $\mathrm{CD} 8^{+}$and $\mathrm{V} Y \mathrm{~V} \delta 2 \mathrm{~T}$ cells in the $\mathrm{CD} 3^{+}$ T cells subsets in those with MVD variants. A possible explanation was that CD $8^{+}$and Vy $9 \mathrm{~V} \delta 2 \mathrm{~T}$ cells might home to the skin in the PK patients with $M V D$ variants. An imbalance in the number and/or function of resident $\alpha \beta$ and $\gamma \delta \mathrm{T}$ cells in the skin has been associated with chronic inflammation and skin-related diseases [10]. A reduction in the number of peripheral $\gamma \delta T$ cells, along with the elevated numbers in the skin, has been reported in patients with psoriasis [11]. As for non-conventional lymphocytes, human $\gamma \delta$ T-cell acts as the first line of defense and bridge the innate and adaptive immune systems, representing $<5 \%$ of peripheral T-cell in the adult human peripheral blood. It is well established that multiple phosphorylated mevalonate metabolites are potent agonists of

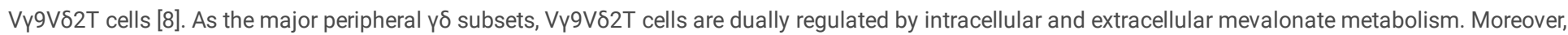
the peripheral Vy9V82 T cells from PK patients with MVD variants showed a higher proportion expressed TNF-a compared with NCs. In a different way, the dysregulated secretion of IFN-y by CD $8+T$ cells were observed in the PK patients with MVK variants. It is noteworthy that the IFN-y signaling for CD ${ }^{+} \mathrm{T}$ cells differentiation are delivered early in the immune response. The autocrine IFN- $\gamma$ signaling plays an important role in Th1 differentiation and CD $8^{+} \mathrm{T}$ cells crosspriming $[10,12,13]$. It suggested that autoreactive $C D 8^{+}$and $y \delta T$ cells might play a critical role in the skin-specific autoinflammatory PK. However, it was not known to what extent, if any, the pro-inflammatory effects of these T cells might affect the pathogenesis of PK.

Danger signals from exogenous pathogens and endogenous keratinocyte death might trigger skin inflammation [14, 15]. Under certain circumstances, genetic defects in mevalonate pathway might block DNA degradation during epidermal cornification and develop a vertical "column" of parakeratosis, histologically defined as a cornoid lamella $(\mathrm{CL})$. In the context of a heterogeneous group of disease, CL unifies all phenotypes of PK. It is remarkable that non-specific papillary dermal lymphocytic infiltration are frequently seen under the CL $[16,17]$. The local immune cell infiltration and chronic activation are involved in the pathomechanisms of PK [18]. The limitations of this study include a small sample size and the lack of comparison of $C D 8^{+} T$ and $y \delta T$ cells in the lesions of PK with $M V K$ or $M V D$ variants. Further investigation is under way to explore T cells in the lesions of PK.

Taken together, our findings showed alterations in peripheral T-cell subsets in PK patients and provided the cues to further studies on autoreactive CD8 $+\mathrm{T}$ and YठT cells in the pathogenesis of autoinflammatory keratinization.

\section{Declarations}

\section{Acknowledgments}

We thank all patients and family members who participated in this study.

\section{Funding}

This work was supported by the grants from National Natural Science Foundation of China (No. 81673073, 81974471).

\section{Availability of data and materials}

All data generated or analyzed during this study are included in this published article and its additional files.

\section{Ethics approval and consent to participate}

This study was approved by the Scientific Ethical Committee of Huashan Hospital, Fudan University (registration no. KY2017-367).

\section{Consent for publication}

Informed consent was obtained from all subjects.

\section{Competing interests}


The authors declare that they have no conflict of interest.

\section{ORCID}

Zhenghua Zhang ${ }^{\mathrm{ID}}$ https://orcid.org/0000-0001-9335-7022

\section{Authors' contributions}

Conception and design by ZHZ and LS. Study materials and patients provided by ZHZ and KXY. Experiments performed by LT and YKH. Data analysis and interpretations by LS, ZHZ, CHL, LT, YKH; The manuscript was written by ZHZ and LT; All aspects of the study were supervised by ZHZ and LS. All authors read and approved the final manuscript.

\section{References}

1. Takeichi T, Akiyama M. Familial or sporadic porokeratosis as an autoinflammatory keratinization disease. J Dermatol. 2019; 46(4):e125-6.

2. Akiyama M, Takeichi T, McGrath JA, Sugiura K. Autoinflammatory keratinization diseases: An emerging concept encompassing various inflammatory keratinization disorders of the skin. J Dermatol Sci. 2018; 90(2):105-11.

3. Akiyama M, Takeichi T, McGrath JA, Sugiura K. Autoinflammatory keratinization diseases. J Allergy Clin Immunol. 2017; 140(6):1545-7.

4. Zhang SQ, Jiang T, Li M, Zhang X, Ren YQ, Wei SC, et al. Exome sequencing identifies MVK mutations in disseminated superficial actinic porokeratosis. Nat Genet. 2012; 44(10):1156-60.

5. Zhang Z, Li C, Wu F, Ma R, Luan J, Yang F, et al. Genomic variations of the mevalonate pathway in porokeratosis. Elife. 2015 ; 4: e06322.

6. Li M, Li Z, Wang J, Ni C, Sun Z, Wilson NJ, et al. Mutations in the mevalonate pathway genes in Chinese patients with porokeratosis. J Eur Acad Dermatol Venereol. 2016; 30(9): 1512-7.

7. Leng Y, Yan L, Feng H, Chen C, Wang S, Luo Y, et al. Mutations in mevalonate pathway genes in patients with familial or sporadic porokeratosis. J Dermatol. 2018; 45(7): 862-6.

8. Thurnher M, Gruenbacher G. T lymphocyte regulation by mevalonate metabolism. Sci Signal. 2015; 8(370): re4.

9. Bekkering S, Arts RJW, Novakovic B, Kourtzelis I, van der Heijden CDCC, Li Y, et al. Metabolic Induction of Trained Immunity through the Mevalonate Pathway. Cell. 2018;172(1-2): 135-46. e9.

10. Sabat R, Wolk K, Loyal L, Döcke WD, Ghoreschi K. T cell pathology in skin inflammation. Semin Immunopathol. 2019; 41(3): $359-77$.

11. Laggner U, Di Meglio P, Perera GK, Hundhausen C, Lacy KE, Ali N, et al. Identification of a novel proinflammatory human skin-homing Vy9V82 T cell subset with a potential role in psoriasis. J Immunol. 2011; 187: 2783-93.

12. de Araújo-Souza PS, Hanschke SC, Viola JP. Epigenetic control of interferon-gamma expression in CD8 T cells. J Immunol Res. 2015; $2015: 849573$.

13. Boehncke WH, Brembilla NC. Autoreactive T-Lymphocytes in Inflammatory Skin Diseases. Front Immunol. 2019; 10 : 1198.

14. Yatim N, Cullen S, Albert ML. Dying cells actively regulate adaptive immune responses. Nat Rev Immunol. 2017; 17(4): 262-75.

15. Eckhart L, Tschachler E. Control of cell death-associated danger signals during cornification prevents autoinflammation of the skin. Exp Dermatol. 2018; 27(8): 884-91.

16. Shumack S, Commens C, Kossard S. Disseminated superficial actinic porokeratosis. A histological review of 61 cases with particular reference to lymphocytic inflammation. Am J Dermatopathol. 1991;13(1): 26-31.

17. Biswas A. Cornoid lamellation revisited: apropos of porokeratosis with emphasis on unusual clinicopathological variants. Am J Dermatopathol. $2015 ; 37(2): 145-55$.

18. Matsuyama K, Mizutani Y, Takahashi T, Shu E, Kanoh H, Miyazaki T, et al. Enhanced dendritic cells and regulatory $\mathrm{T}$ cells in the dermis of porokeratosis. Arch Dermatol Res. 2017; 309(9): 749-56.

\section{Figures}




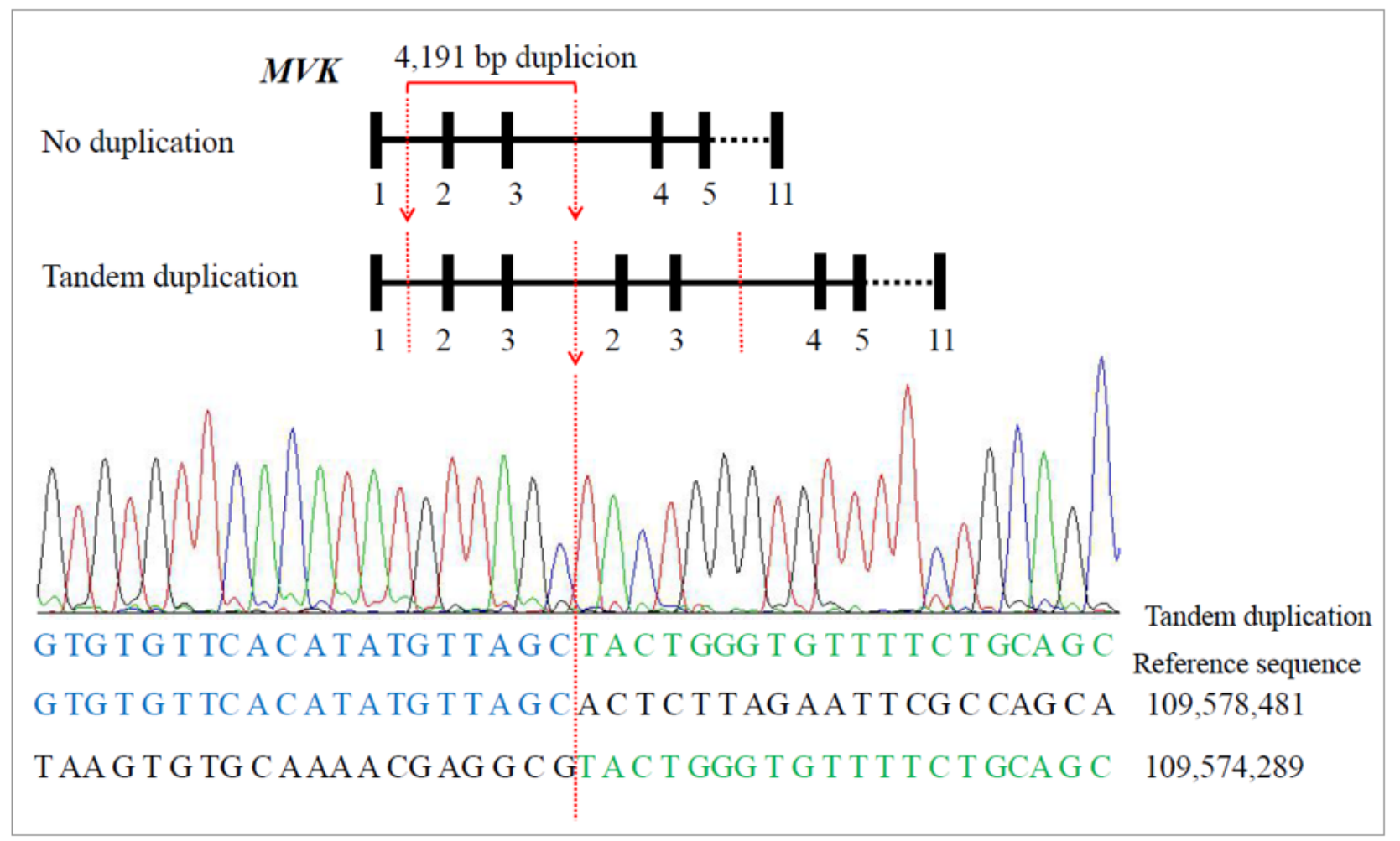

Figure 1

Schematic of a mutation c.118_226+1337dup in MVK by CNVplex assay.
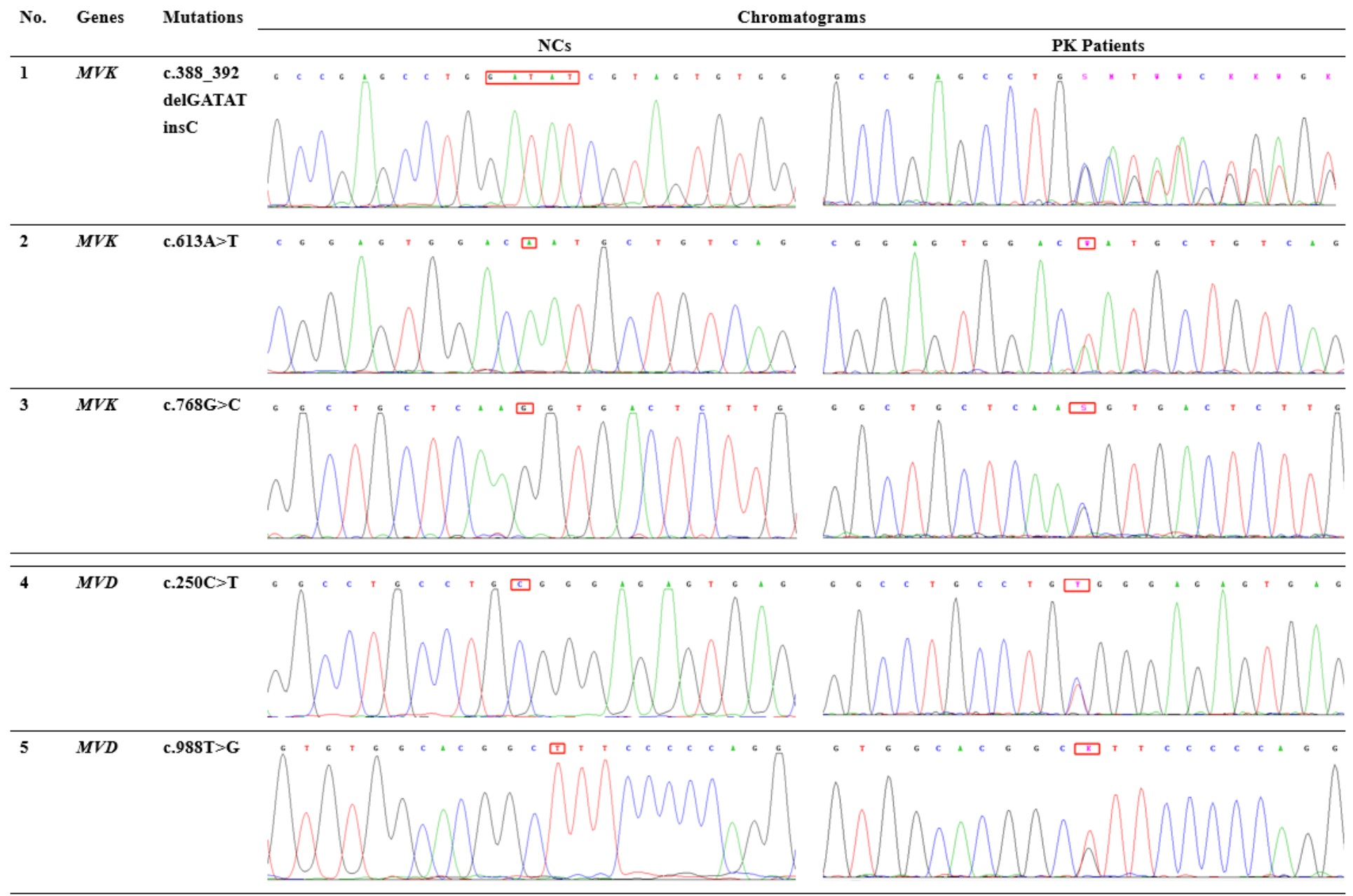
Figure 2

Sanger sequencing chromatograms of NCs and PK patients at 5 novel mutation sites in MVK and MVD.

A
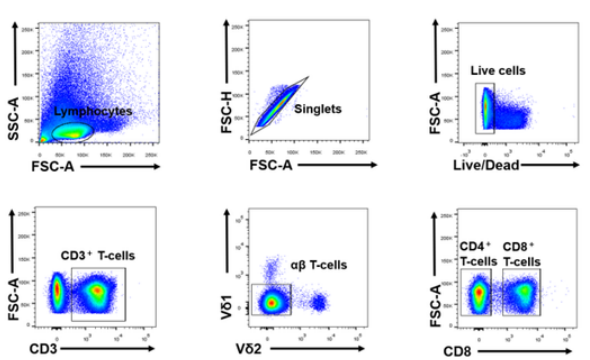

B

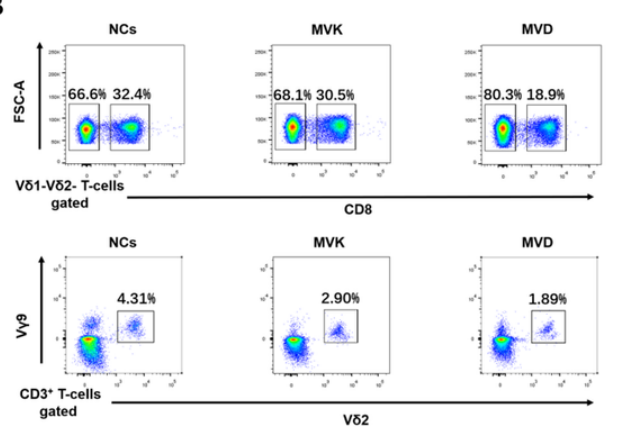

C
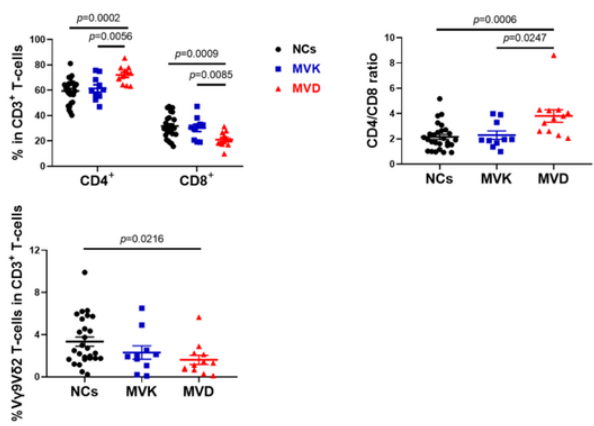

Figure 3

Gating strategy for flow cytometry (A), representative flow cytometry analyses (B) and scatterplot graphs (C) showed the frequencies of CD4+, CD8+ and Vy9V82 T cells in the CD3+ T-cell subsets and the ratio of CD4+T-cell/CD8+T-cell. Notes: MVK: the PK patients with MVK variants ( $n=10$ ); MVD: PK patients with MVD variants $(n=12)$; NCs: normal controls $(n=27)$. 
A
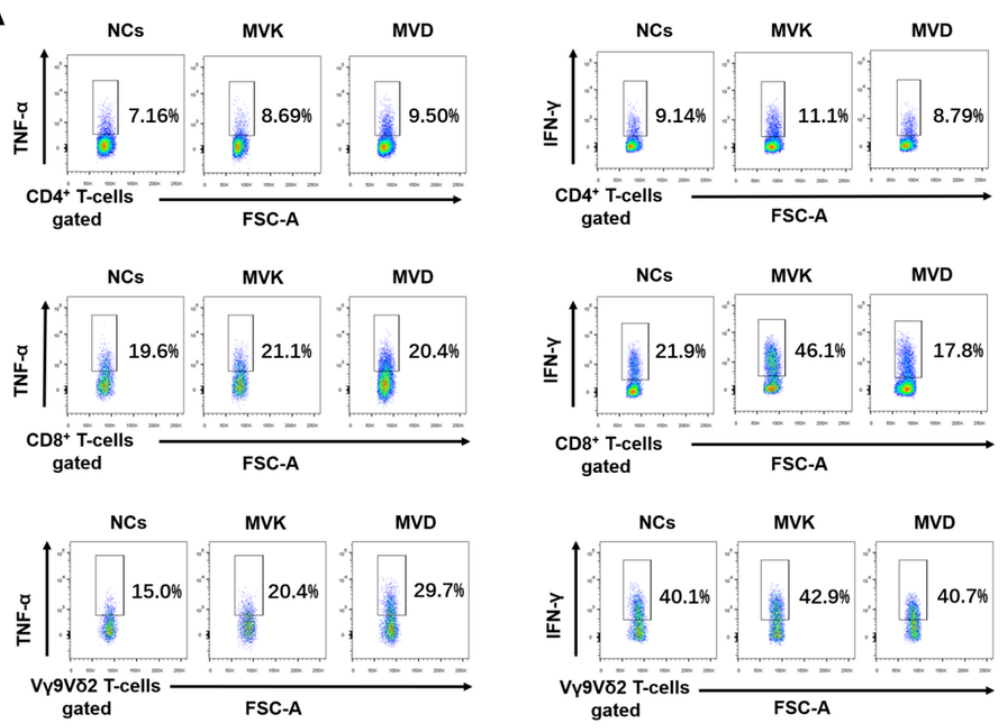

B
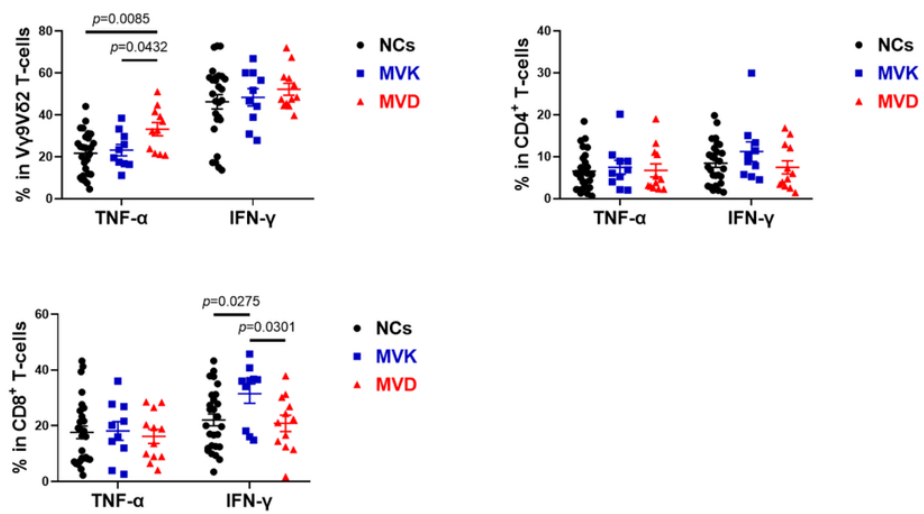

\section{Figure 4}

Representative flow cytometry analyses (A) and scatterplot graphs (B) of TNF- $a$ and IFN-y production in CD4+, CD8+, and Vy9V82 T cells in the PK patients with MVK and MVD variants. Notes: MVK: the PK patients with MVK variants $(n=10) ;$ MVD: PK patients with MVD variants $(n=12) ;$ NCs: normal controls $(n=$ 27).

\section{Supplementary Files}

This is a list of supplementary files associated with this preprint. Click to download.

- Additionalinformation.docx 